\title{
An Improved Design of L-probe Feeding Microstrip Antenna Array for 4G Applications
}

\author{
He Wang ${ }^{1, ~ a ~}{ }^{*}$, Hu Yang ${ }^{2, b}$, Lei Gu ${ }^{3, c}$, Fei Zhao ${ }^{4, d}$, Wenlu Yi,e \\ ${ }^{1}$ National University of Defense Technology, Changsha, China \\ ${ }^{2}$ National University of Defense Technology, Changsha, China \\ 3,4,5Southwest Electronics and Telecommunication Technology Research Institute, Chengdu, China \\ aiamkennan@163.com, byanghu90@163.com, ’gulei100121@sina.com@163.com \\ dfeizhao1983@hotmail.com, èyinwl@sina.com
}

Keywords: microstrip antenna, broadband, wide beamwidth, high gain, 4G mobile communication systems.

\begin{abstract}
To meet the high requirements of $4 \mathrm{G}$ mobile communication systems, such as broadband, wide beamwidth and high gain, an improved microstrip antenna array fed by L-probe is proposed. Generally, most of the $4 \mathrm{G}$ antenna array elements have wide band and a gain of $3-5 \mathrm{dBi}$. But the beamwidth in the horizontal plane is narrow, which is less than $70^{\circ}$ usually. To solve this problem, an improved design of the patch ground is introduced. Then an improved patch antenna and its array are designed. The simulated results show that the return loss of the improved patch is less than -10dB from $1.88 \mathrm{GHz}$ to $2.65 \mathrm{GHz}$, which has a complete coverage of TD-LTE $4 \mathrm{G}$ band. And the beamwidth expands to almost $120^{\circ}$ at the same time. Though the gain of the patch is only above $5 \mathrm{dBi}$, a $1 \times 4$ antenna array can get a gain of $11.85 \mathrm{dBi}$ at the center frequency. Therefore, the proposed array is more profitable for the mobile communication systems.
\end{abstract}

\section{Introduction}

With the development of mobile communication systems, the characteristics of antenna are attached much more importance, such as size, impedance bandwidth, gain and beamwidth. According to the features of low profile, light weight and the conformity to mounting structures, patch antennas are the prior option to tackle the size problem [1]. As the evolution of 3G mobile networks, TD-LTE is the primary technology behind 4G [2]. Recently, operating permission for TD-LTE was awarded by Chinese government to the three major mobile operators in China. China Mobile has been authorized to operate TD-LTE on three frequency bands- $1880-1900 \mathrm{MHz}, 2320-2370 \mathrm{MHz}$ and $2575-2635 \mathrm{MHz}$. China Unicom obtains two bands-they are 2300-2320MHz and 2555-2575MHz. For China Telecom, it has a dual band of $2370-2390 \mathrm{MHz}$ and $2635-2655 \mathrm{MHz}$ [3]. However, the bandwidth of traditional patch antennas is too narrow to cover the whole TD-LTE frequency band, so the monopole planar antennas are proposed [4]. In general, their gain is about $3 \mathrm{dBi}$, and the beamwidth is up to $70^{\circ}$. In order to realize the omnidirectional radiation in the horizontal plane, more than six antenna elements are needed. Therefore, antennas with a wide beamwidth can decrease the number of element to some extent. Then the patch antenna fed by L-probe is put forward [5]. Owing to its simple structure, broadband, moderate gain and wide bandwidth [6], it is quite suitable for the $4 \mathrm{G}$ mobile communication applications. 
In this paper, we put forward a patch antenna element fed by L-probe. The frequency range $\left(\left|S_{11}\right|<-10 \mathrm{~dB}\right)$ of the designed antenna covers the $4 \mathrm{G}$ band of TDD-LTE. Then the element is improved to obtain a broader beamwidth of $114^{\circ}$. And finally, a $1 \times 4$ array is designed to achieve a higher gain of $11.85 \mathrm{dBi}$, which can meet the demand for broadband, wide beamwidth and high gain in the mobile communication systems.

\section{The Design of L-probe Feeding Microstrip Patch}

As shown in Fig. 1, a conventional L-probe feeding microstrip patch consists of three components.

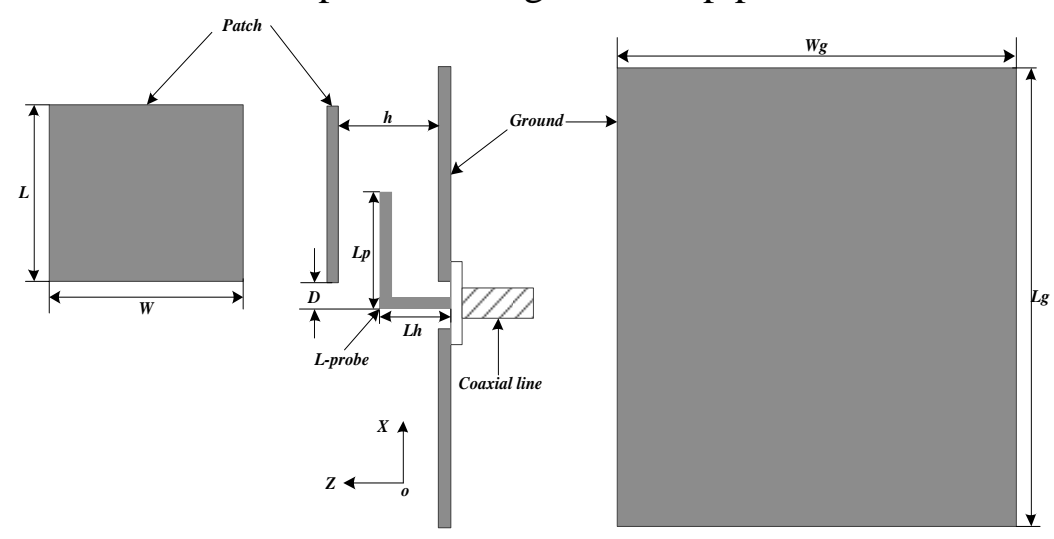

Fig. 1 Geometry of the conventional patch

On the top of the antenna is a rectangular radiation patch, which is fed by an L-shaped coaxial probe, so the normal $\mathrm{TM}_{01}$ mode of the patch can be excited. The size of the patch is about half of the wavelength. At the bottom of the antenna is the metallic ground plane. And the feeding probe penetrates through the ground by a hole. Between the rectangular radiation patch and the ground is filled with air, which may broaden the bandwidth. We can adjust the size of the probe and the height of the air layer to get a wide bandwidth. For $4 \mathrm{G}$ applications the optimized parameters of this antenna are displayed in Table 1.

Table 1 The optimized parameters of the conventional patch

\begin{tabular}{|c|c|c|c|c|c|c|c|c|}
\hline Parameter & $L$ & $W$ & $L g$ & $W g$ & $h$ & $L p$ & $L h$ & $D$ \\
\hline Value(mm) & 51.1 & 61.3 & 245.4 & 204.5 & 13.5 & 23.5 & 10.5 & 5.1 \\
\hline
\end{tabular}

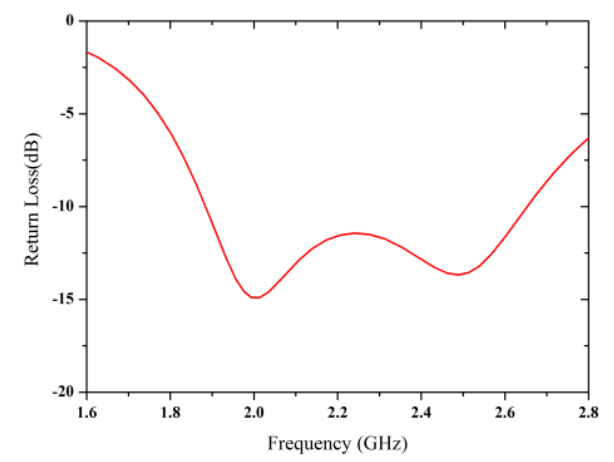

Fig. 2 Simulated return loss of the conventional patch

Fig. 2 shows the return loss of the conventional L-probe feeding microstrip patch. And the return loss is less than $-10 \mathrm{~dB}$ from $1.88 \mathrm{GHz}$ to $2.66 \mathrm{GHz}$, which fully covers the TD-LTE $4 \mathrm{G}$ band $(1.88 \mathrm{GHz}-2.655 \mathrm{GHz})$. And the fractional bandwidth is $34.36 \%$. Because the capacitance introduced by the L-probe suppresses some of the inductance brought by the feed probe, the wide bandwidth is obtained. 
The simulated radiation patterns of the antenna at $1.9 \mathrm{GHz}, 2.25 \mathrm{GHz}$ and $2.6 \mathrm{GHz}$ are shown in Fig. 3 , Fig. 4 and Fig. 5, respectively.

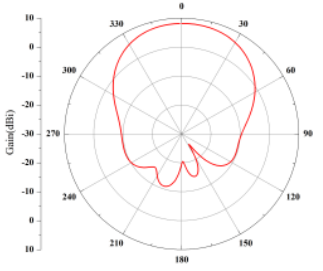

(a) XOZ $€$ plane

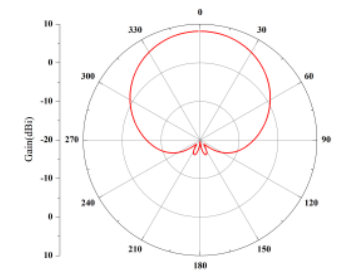

(b) XOY $(\mathrm{H})$ plane

Fig. 3 Simulated radiation pattern at $1.9 \mathrm{GHz}$

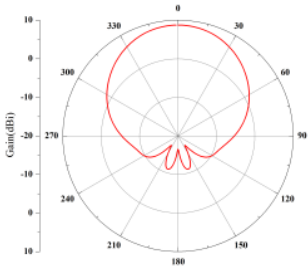

(a) XOZ $€$ plane

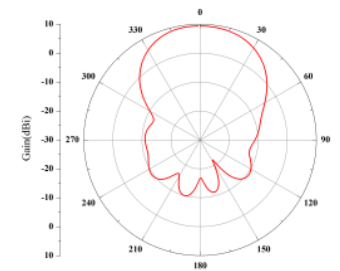

(a) XOZ $€$ plane

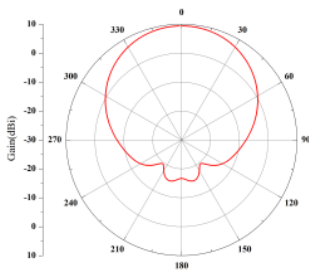

(b) XOY (H) plane

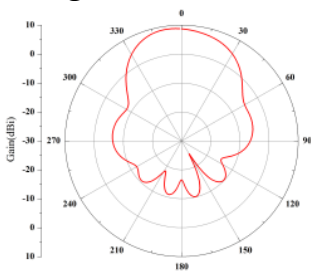

(b) XOY (H) plane

Fig. 5 Simulated radiation pattern at $2.6 \mathrm{GHz}$

As can be seen, the beamwidth of $\mathrm{H}$-plane is about $70^{\circ}$, which is almost not changed through the whole band. And the beamwidth of E-plane varies from about $70^{\circ}$ to $50^{\circ}$, when the frequency changes from $1.9 \mathrm{GHz}$ to $2.6 \mathrm{GHz}$. Besides, the beamwidth of $\mathrm{H}$-plane is always a little wider than that of E-plane. If an omnidirectional pattern in the horizontal plane is required by this antenna, 5 or 6 antennas should be used. The gain is above $7.5 \mathrm{dBi}$ over $1.88 \mathrm{GHz}-2.65 \mathrm{GHz}$. Moreover, the maximum gain is $9.2 \mathrm{dBi}$, which happens at $2.22 \mathrm{GHz}$.

\section{The Design of The Improved Antenna}

\section{A. An improved L-probe feeding microstrip patch}

Generally, to reduce the complexity of the mobile communication system, the antennas need to own the feature of wide beamwidth in the horizontal plane. Unfortunately the beamwidth of the conventional antenna can't satisfy this requirement in many cases. Through the study of all parameters designed in part I , it is found that we can realize the purpose of wide beamwidth by only adjusting the size of the ground. Therefore, an improved antenna is proposed, which structure is identical to the conventional patch as shown in Fig.1. And the optimized parameters of the improved antenna are displayed in Table 2 .

Table 2 The optimized parameters of the improved patch

\begin{tabular}{|c|c|c|c|c|c|c|c|c|}
\hline Parameter & $L$ & $W$ & $L g$ & $W g$ & $h$ & $L p$ & $L h$ & $D$ \\
\hline Value(mm) & 51.1 & 61.4 & 245.4 & 28.0 & 13.5 & 23.5 & 10.5 & 5.1 \\
\hline
\end{tabular}

Fig. 6 shows the return loss of the improved L-probe feeding microstrip patch. And the return loss is less than $-10 \mathrm{~dB}$ from $1.82 \mathrm{GHz}$ to $2.68 \mathrm{GHz}$, which fully covers the TD-LTE $4 \mathrm{G}$ band $(1.88 \mathrm{GHz}-2.655 \mathrm{GHz})$ too. And the fractional bandwidth is $38.22 \%$, which is $3.86 \%$ wider than the conventional patch.
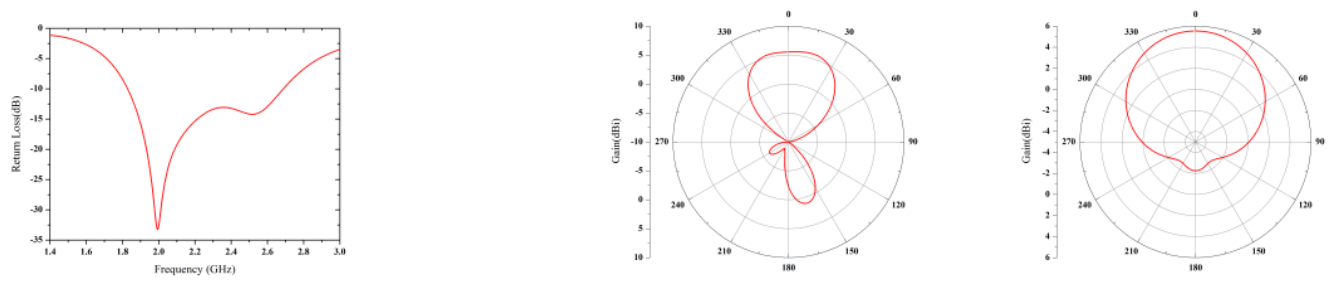


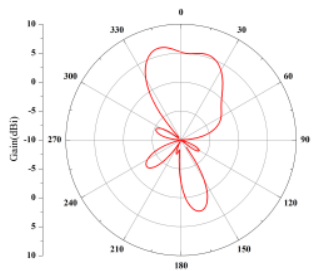

(a) XOZ (E) plane

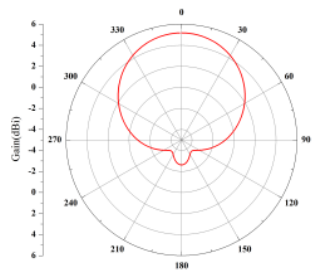

(b) XOY (H) plane

Fig. 7 Simulated radiation pattern at $1.9 \mathrm{GHz}$

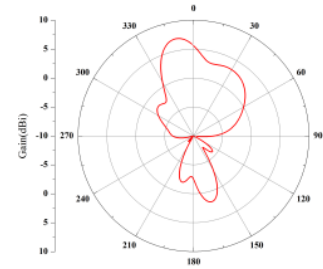

(a) XOZ (E) plane

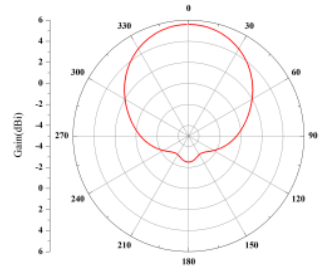

(b) XOY (H) plane

Fig. 8 Simulated radiation pattern at $2.25 \mathrm{GHz}$ Fig. 9 Simulated radiation pattern at $2.6 \mathrm{GHz}$ Fig. 7-Fig. 9 show the radiation pattern of the improved L-probe feeding patch at $1.9 \mathrm{GHz}, 2.25 \mathrm{GHz}$ and $2.6 \mathrm{GHz}$, respectively.

As can be seen, the beamwidth of H-plane is obviously expanded, which is almost $120^{\circ}$ in the whole band. So we can use only three antennas of the improved type to get an omnidirectional coverage in the horizontal plane. The amount of antennas is half of the conventional form, which is very attractive in engineering. Compared with the conventional form, the gain of the improved antenna decreases about $3 \mathrm{dBi}$. This is mainly because the H-plane beamwidth of the improved antenna is about two times than the former. But the gain of the improved patch is still above $5 \mathrm{dBi}$ in the whole band, which is higher than most common base-station antennas.

\section{B. An array of the improved patch}

To further verify the wide beamwidth performance of the improved patch and considering the need of high gain in actual system, a $1 \times 4$ antenna array is also simulated. As shown in Fig. 10, the spacing between adjacent antenna elements is $67 \mathrm{~mm}$ to avoid grating lobe at high frequency. To maintain the wideband property, the antenna elements are fed by an equal-power splitter.

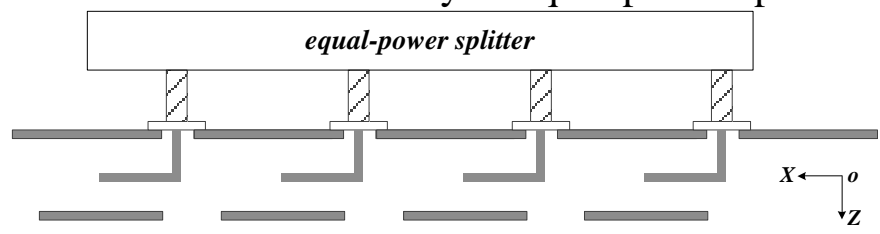

Fig. 10 Geometry of the $1 \times 4$ antenna array

The return loss can fully cover the TD-LTE $4 \mathrm{G}$ band $(1.88 \mathrm{GHz}-2.655 \mathrm{GHz})$ as well. Here we focus on the pattern feature. Fig.11-Fig.13 shows the simulated radiation patterns of the antenna array at $1.9 \mathrm{GHz}, 2.25 \mathrm{GHz}$ and $2.6 \mathrm{GHz}$.

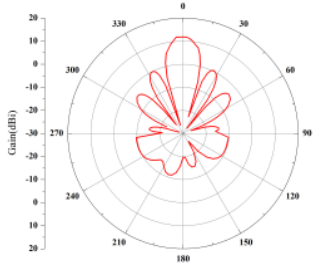

(a) XOZ (E) plane

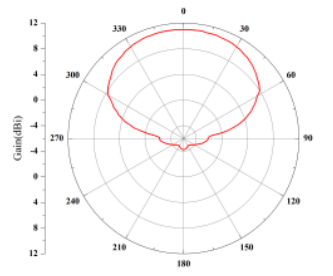

(b) XOY (H) plane

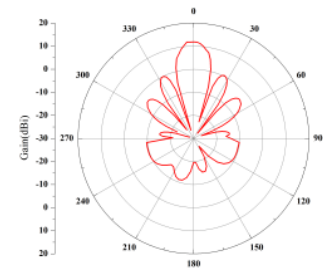

(a) XOZ (E) plane

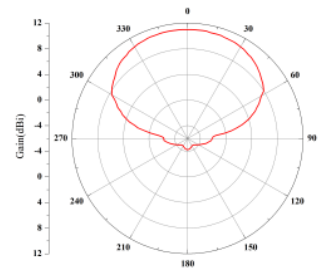

(b) XOY (H) plane

Fig. 11 Simulated radiation pattern at $1.9 \mathrm{GHz}$
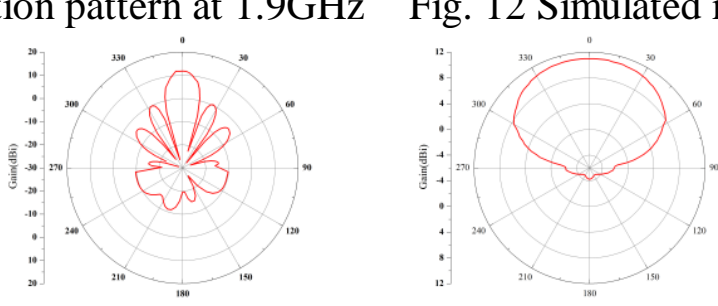

(a) XOZ (E) plane

(b) XOY $(\mathrm{H})$ plane

Fig. 13 Simulated radiation pattern at $2.6 \mathrm{GHz}$ 
The beamwidth of the H-plane keeps wide, which is still about $120^{\circ}$. The gain is $11.85 \mathrm{dBi}$ at the center frequency $2.25 \mathrm{GHz}$. As a result, the gain of the antenna is improved without affecting the bandwidth and beamwidth character.

\section{Summary}

The ideal characteristic of the base-station antenna is to have broadband, high gain and wide beamwidth at the same time. However these three characteristics are contradictory. To tackle the problem, an improved L-probe feeding patch antenna is proposed. Compared with other work, the proposed antenna broadened beamwidth without affecting the wide band character. Though the gain is not high, it can be improved by antenna array. And the simulated results of a $1 \times 4$ array have demonstrated it. With the benefits of wide beamwidth, the mobile communication system can be simpler and the cost of the system will also be reduced. These make our proposed antenna more attractive in $4 \mathrm{G}$ applications.

\section{References}

[1] Y.X. Guo, K.M. Luk and K.F. Lec:'L-probe proximity-fed short-circuited patch antennas', Electronic Letters, 25th November 1999, Vol.35, pp.2069-2070.

[2] Vallecchi A, Gentili G.B.'Design of dual- polarization series-fed microstrip arrays with low losses and high polarization purity [J]. IEEE Trans. Antennas Propag., 2005, 53(5), pp. 1791-1798.

[3] Le Kuai, Xiaoxing Yin, Xiaowei Zhu, Chanyuan Chen: A Wide-band Dual Polarization Microstrip Antenna for TD-LTE Applications, IEEE, 2014, AP-S, pp. 1843-1844.

[4] F. Guan, X. Luo, and X. Lin. A Novel Wideband Dual-Polarized Dipole Antenna[C]. in The Proceedings of the Second International Conference on Communications, Signal Processing, and Systems, 2014, 847-852.

[5] Yongxin Guo, Michael Yan Wah Chia, Zhi Ning Chen and Kwai-Man Luk: 'Wide-Band L-Probe Fed Patch Antenna for Conial-Pattern Radiation'. IEEE Trans. Antennas Propag., 2004, VOL52, NO4, pp. 1115-1116.

[6] Lei Wang, Yongxin Guo, Weixing Sheng: 'Wideband High-Gain 60-GHz LTCC L-Probe Patch Antenna Array With a Soft Surface'. IEEE Trans. Antennas Propag., 2013, VOL61, NO4, pp. 1802-1808. 